\title{
Standard Model Bosons as Composite Particles
}

D. E. Kahana

Continuous Electron Beam Accelerator Facility

12000 Jefferson Avenue, Newport News, VA 23606

S. H. Kahana

Physics Department, Brookhaven National LaborRgey
Upton, NY 11973

OCT $O 41990$

\section{Continuous}

Electron

BEAM

Accelerator

FACILITY

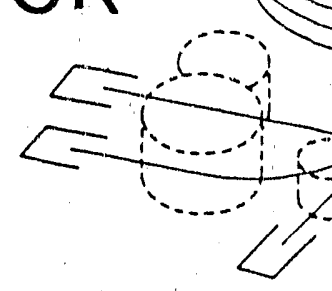

SURA SOUTHEASTERN UNVERSTTES REEAACCH ASSOCLITION

\section{CEBAF}

Newport News, Virginia 
Copies available from:

\author{
Library \\ CEBAF \\ 12000 Jefferson Avenue \\ Newport News \\ Virginia 23606
}

The Southeastern Universities Research Association (SURA) operates the Continuous Electron Beam Accelerator Facility for the United States Department of Energy under contract DE-AC05-84ER40150.

\title{
DISCLAIMER
}

This report was prepared as an account of work sponsored by the United States government. Neither the United States nor the United States Department of Energy, nor any of their employees, makes any warranty, express or implied, or assumes any legal liability or responsibility for the accuracy, completeness, or usefulness of any information, apparatus, product, or process disclosed, or represents that its use would not infringe privately owned rights. Reference herein to any specific commercial product, process, or aervice by trade name, mark, manufacturer, or otherwise, does not necessarily constitute or imply ita endorsement, recommendation, or favoring by the United States government or any agency thereof. The views and opinions of authors expressed herein do not necessarily state or reflect those of the United States government or any agency thereof. 
PPT - \#\#\#

\title{
Standard Model Bosons as Composite Particles
}

\author{
D. E. Kahana ${ }^{1}$ and S. H. Kahana ${ }^{2}$ \\ CEBAF, 12000 Jefferson Ave. \\ Newport News, VA 23606 \\ and \\ Physics Department, BNL, \\ Upton, NY 1.1973
}

\begin{abstract}
The Standard model of electro-weak interactions is derived from a Nambu, Jona-Lasinio type four-fermion interaction, which is assumed to result from a more basic theory valid above a very high scale $\Lambda$. The masses of the gauge bosons and the Higgs are then produced by dynamical symmetry breaking of the Nambu model at an intermediate scale $\mu$, and are evolved back to experimental energies via the renormalisation group equations of the Standard model. The weak angle $\sin ^{2}\left(\theta_{W}\right)$ is predicted to be $\frac{3}{8}$ at the scale $\mu$, as in grand unified theories, and is evolved back to the experimental value at scale $M_{W}$, thus determining $\mu \sim 10^{13} \mathrm{GeV}$. Predictions for the ratios of the masses of the gauge and the Higgs bosons to the top quark mass, at experimental energies, are also obtained.
\end{abstract}

${ }^{1}$ DOE funded Research at CEBAF, 12000 Jefferson Ave., Newport News, Virginia 23606

${ }^{2}$ Supported in part by USDOE contract DE-AC02-76CH00016. 


\section{Introduction}

Recently, in very interesting works, Nambu [1], as well as Bardeen, Hill and Lindner [2], and others [3], have suggested replacing the Higgs mechanism [4] in the Standard model [5] with a dynamical symmetry breaking approach generated by four-fermion interactions of the top quark. In fact, their model for the Higgs sector is that of Nambu and Jona-Lasinio [6], and one may recover the usual Higgs meson as a composite of $t$ and $\bar{t}$. One of the present authors as well as others [7] have followed the original NJL work by introducing vector as well as scalar four fermion interactions, thereby also generating vector mesons as composite fields in this model. Here we attempt to combine these two approaches, to generate the entire electro-weak interaction from a four-fermion effective lagrangian. The $W, Z$ and Higgs then appear as coherent composites of all quarks and leptons, not just the heaviest quark. There are clear problems with such an approach which will become evident, but there are also possible rewards.

The effective four fermion theory of this paper is assumed to be the correct interaction at some high scale $\Lambda$, and could presumably be obtained as the low energy limit of some more basic theory, applicable at even higher energy; for instance by integrating out the high energy non-fermionic degrees of freedom above the scale $\Lambda$. Subsequently, we integrate the single fermion loops of the four-fermion theory to obtain an effective action at an intermediate scale $\mu$. We find that the meson sector of the theory induced by the NLJ model at the scale $\mu$ can be, under certain restrictive conditions, cast into the form of the $S U_{L}(2) \times U_{R}(1)$ Standard model. Below the scale $\mu$ the effects of the extended $S U(3) \times S U(2) \times U(1)$ interactions are included through the usual renormalisation group equations. This parallels the method of reference [2]; they differ in performing a matching at the scale $\Lambda$, where the boson wave function renormalisations vanish, through compositeness conditions. As a caveat we must add that this procedure is clearly sornewhat arbitrary for the non-renormalisable NJL model, but like [2], we suppose that some limited degree of fine-tuning eliminates the quadratic divergences from the theory and leaves logarithmic divergences which can be handled in the renormalisation group scheme.

The masses and mixing angles for the composite particles are then obtained at an intermediate but nevertheless still large scale $\mu$ in our approach. For example, $\sin ^{2}\left(\theta_{W}\right)$ is found to be a, simple rational number, reminiscent of grand unified models, and in addition the $W$ and $Z$ masses are very simply related to the top quark mass. Several authors, in particular Marciano [9] and Nambu [1] have pointed out that one mechanism probably generates mass for both the top quark and the massive vector bosons, since the $W, Z$ and top all seem to lie at more or less the same scale. Since, as we show, the standard electroweak theory is 
recovered at the intermediate scale $\mu$, by imposing what seem to be necessary consistency conditions on the effective interaction for the composite Higgs and vector bosons, we assume that the masses, etc., may be taken as boundary conditions [2] for the standard model renormalisation group equations [10], and evolved downward as usual, to yield a determination of $\mu$ and a prediction of the ratio $\frac{M_{t}}{M_{w}}$ at present experimental energies. The indefiniteness of the scales $\Lambda$ and $\mu$, and the direct use of the parameters of the effective lagrangian for the composites as boundary conditions of course is reflected in a similar indefiniteness of the predictions we make.

We note that although we have not attempted to include the strong interactions explicitly here, it is clear that one could generate composite gluons and massive vector bosons at the same time in an extended version of this same scheme.

\section{The Model}

The model is defined by:

$$
L=\bar{\psi} i \not \partial \psi-\frac{1}{2}\left[\left(\bar{\psi} G_{S} \psi\right)^{2}-\left(\bar{\psi} G_{s} \tau \gamma_{5} \psi\right)^{2}\right]-\frac{1}{2} G_{B}^{2}\left(\bar{\psi} \gamma_{\mu} Y \psi\right)^{2}-\frac{1}{2} G_{W}^{2}\left(\bar{\psi} \gamma_{\mu} \tau P_{L} \psi\right)^{2}
$$

Here $\psi=\left[\psi_{i}\right]$ is a column vector: $i=\left\{t_{L}, t_{R}, b_{L}, b_{R}, e_{L}, e_{R}, \nu_{L}\right\}$, and this pattern is of course duplicated for each generation of fermions that is present. $G_{B}$ and $G_{W}$ are universal couplings for the isoscalar and isovector interactions respectively, while $Y$ and $\tau$ are defined in the usual fashion. $P_{L}=\frac{1}{2}\left(1-\gamma_{5}\right)$ is the projector on left-handed fermions. Breaking of weak isospin symmetry is introduced via the scalar coupling $G_{S}$, which is taken to be a diagonal matrix; we could of course, as suggested in reference [2], incorporate Cabibbo-like mixings between the generations by allowing $G_{S}$ also to have off-diagonal elements, but this is an added complication which we omit for now. We are also employing an Euclidean metric with $\gamma_{\mu}{ }^{2}=-1$. The scalar-pseudoscalar interaction above has been treated in a somewhat cavalier fashion, which however has no profound consequences for what follows. The choice made in equation (2.1) would seem to be unique if the standard model for the quark-boson interactions is to be recovered, and could be derived for instance by the introduction of very massive vector bosons $\mathbf{W}_{\mu}^{k}, \mathbf{B}_{\mu}$ at scale $\Lambda$. The usual $W$ and $Z$ then arise from the lagrangian in $\mathrm{Eq}(2.1)$, as much lighter composite images. The couplings $G_{S}, G_{B}, G_{W}$ have dimensions $[L]^{-1}$ as required by the four fermion coupling in $d=4$, and are later demonstrated to be inversely proportional to the cutoff scale $\Lambda$. As in references [11] we consiruct the meson lagrangian using the methods of Gross and Neveu. [12]. A uxiliary fields $F \equiv\left[\sigma, \pi^{i}, B_{\mu}, W_{\mu}^{k}\right]$ 
are introduced into the partition function:

$$
\begin{aligned}
Z(J) & =\int \mathrm{D}[F] \int \mathrm{D} \psi \mathrm{D} \bar{\psi} \exp \left\{-\int \mathrm{d}^{4} x\left(L+L_{c l}(F)\right)+J[\bar{\psi} \psi]\right\} . \\
L_{c l} & =\frac{1}{2}\left(\sigma^{2}+\left(\pi^{i}\right)^{2}\right)+\frac{1}{2}\left(B_{\mu}^{2}+\left(W_{\mu}^{k}\right)^{2}\right)+J F
\end{aligned}
$$

Shifting the fields according to:

$$
\begin{array}{cc}
\sigma=\sigma^{\prime}+\bar{\psi} G_{S} \psi+J_{S} / G_{S}, & B_{\mu}=B^{\prime}{ }_{\mu}+G_{B} \bar{\psi} \gamma_{\mu} Y \psi+J_{B} / G_{B}, \\
\pi=\pi^{\prime}+i \bar{\psi} G_{S} \gamma_{5} \psi+J_{\pi} / G_{S}, & W_{\mu}^{k}=W_{\mu}^{\prime k}+G_{W} \bar{\psi} \gamma_{\mu} \tau^{k} P_{L} \psi+J_{W} / G_{W}
\end{array}
$$

then cancels the four fermion interactions in favour of a coupling between the auxiliary fields and the fermions. The partition function is then essentially that for the coupled mesonfermion theory. We note that at this level gauge invariance is broken only by the quadratic classical terms $L_{c l}$ for the auxiliary fields. The lagrangian for the shifted fields (in which primes may be dropped) then reads:

$$
\begin{gathered}
L^{\prime}=\bar{\psi} \Delta \psi+\frac{1}{2}\left(\sigma^{2}+\left(\pi^{i}\right)^{2}\right)+\frac{1}{2} B_{\mu}^{2}+\frac{1}{2}\left(W_{\mu}^{k}\right)^{2} \\
\Delta=i \gamma_{\mu} D_{\mu}+G_{S}\left(\sigma+i \pi \cdot \tau \gamma_{5}\right)
\end{gathered}
$$

where $D_{\mu}$ is the covariant derivative:

$$
D_{\mu}=\partial_{\mu}-i G_{B} B_{\mu} Y-i G_{W} W_{\mu} \cdot \tau P_{L}
$$

The composite bosons, their propagators and their interactions are obtained by examining the one fermion loop effective action:

$$
\begin{aligned}
I_{\text {eff }} & =I_{\text {class }}-\left(\operatorname{Tr} \ln \Delta-\operatorname{Tr} \ln \Delta_{0}\right) \\
& =I_{\text {class }}-\operatorname{Tr} \ln \left[1+\frac{1}{i \partial \hat{\phi}+M}\left(\tilde{\Omega}_{S}+\Omega_{B}+\Omega_{W}\right)\right]
\end{aligned}
$$

in which:

$$
\Omega_{S}=\Sigma+i \Pi \cdot \tau \gamma_{5}=M+\left(\tilde{\Sigma}+i \tilde{\Pi} \cdot \tau \gamma_{5}\right)
$$

and we have defined scalar fields with the usual dimension by absorbing the coupling $G_{S}$ :

$$
\Sigma=G_{S} \sigma, \quad \Pi^{k}=G_{S} \Pi^{k}
$$

Since $G_{S}$ is a matrix, $\Pi \cdot \tau$ must be symmetrised on $\Pi$ and $\tau$. We have generated an expansion of the action by introducing a scalar meson condensate $\langle\sigma\rangle=\sigma_{0}$ and $G_{S} \sigma_{0}=M$, so that $\Delta_{0}=i \not+M$ is the Dirac operator for massive quarks propagating in the condensed vacuum. 
The model so far is a normal theory of fermions and bosons, manifestly gauge invariant in the fermion-boson sector, but lacking kinetic terms for both scalar and vector bosons. The classical 'hard' mass terms in $\mathrm{Eq}_{\mathrm{q}}(2.6)$ seem to violate gauge invariance at this point, but we will deal with this matter later.

\section{The Boson Action.}

The masses and kinetic energies of all mesons may be generated naturally from $\mathrm{Eq}(2.6)$. For example in the purely scalar sector, eliminating linear terms in the fluctuation of $\sigma$ around the condensate leads to the (NJL) gap equation:

$$
\operatorname{Tr}\left[\frac{1}{i \not \partial+M} G_{S}^{2}\right]=1
$$

and the second order action in $\Omega_{S}$ contains the kinetic energies and masses for the composite scalar:

$$
\begin{aligned}
I_{S} & =\frac{1}{2} \operatorname{Tr}\left[\frac{1}{i \not \partial+M} \Sigma \frac{1}{i \not \partial+M} \Sigma\right]+\frac{1}{2} \sigma^{2} \\
& =\frac{1}{2} \operatorname{Tr}\left\{\left[\frac{\partial^{2}}{\left(-\partial^{2}+M^{2}\right)^{2}}+\frac{M^{2}}{\left(-\partial^{2}+M^{2}\right)^{2}}\right] \Sigma^{2}+\frac{1}{2} \frac{\partial^{2}}{\left(-\partial^{2}+M^{2}\right)^{2}}\left(\partial_{\mu} \Sigma\right)^{2}+\cdots\right\}
\end{aligned}
$$

Third and fourth order terms complete the rest of the Higgs lagrangian. In equation (3.2) we have dropped terms with more than two derivatives of the fields, and we will follow this procedure throughout the paper. It emerges in any case that these terms are of non-leading order in the limit that the scale $\Lambda$ is very large. The scales $\Lambda$ and $\mu$ enter the problem as upper and lower limits for the loop integration in (3.1), which is then given explicitly by:

$$
\frac{1}{4 \pi^{2}} \sum_{i} n_{i} G_{i}^{2} \int_{\mu^{2}}^{\Lambda^{2}} \frac{k^{2} \mathrm{~d} k^{2}}{k^{2}+m_{i}^{2}}=\frac{1}{4 \pi^{2}} \sum_{i} n_{i} G_{i}^{2}\left[\left(\Lambda^{2}-\mu^{2}\right)-m_{i}^{2} \ln \left(\frac{\Lambda^{2}+m_{i}^{2}}{\mu^{2}+m_{i}^{2}}\right)\right]=1 .
$$

Combining (3.3) with (3.2) then cancels the 'hard' (proportional to $\Lambda^{2}$ ) mass terms for the scalar field, leaving only terms which vanish in the limit of no dynamical symmetry breaking, that is, when $\sigma_{0}=0$. Assuming that the top quark dominates, $m_{t}^{2}>m_{i}^{2},(3.3)$ becomes a condition on the top quark coupling $[1,2]$ :

$$
G_{t}^{2}=\frac{4 \pi^{2}}{n_{c}\left(\Lambda^{2}-m_{t}^{2} \ln \left(\frac{\Lambda^{2}}{m_{t}^{2}}+1\right)\right)}
$$

with the colour degeneracy $n_{c}=3$. This fine tuning eliminates the $\Lambda^{2}$ singularities from the (NJL) theory, as Bardeen et. al. remark. A similar fine tuning, or consistency condition, will 
need to be imposed in the vector boson sector to guarantee gauge invariance and the strict vanishing of the photon mass. The kinetic terms for the Higgs may be put into normal form by a wavefunction renormalisation:

$$
\begin{aligned}
& \sigma^{\prime}=Z_{S}^{1 / 2} \sigma_{;} \quad \sigma_{0} \equiv v=Z_{S}^{1 / 2} \sigma_{0}, \\
& Z_{S}^{1 / 2}=\frac{1}{2} \operatorname{Tr}\left[\left(\frac{1}{-\partial^{2}+M^{2}}\right)^{2} G_{S}^{2}\right]
\end{aligned}
$$

V'e note here that this procedure is not without ambiguity [2]. We will simply take the approach in this paper that neither the couplings nor the masses can be unambiguously identified. This will not however prevent us from making at least suggestive predictions. as the ratios of masses and of couplings can be identified unambiguously. Assuming a conventional normalisation for the scalar kinetic terms we find the standard NJL expression for the mass of the Higgs scalar;

$$
\begin{aligned}
& m_{H}^{2}(\mu)=\frac{\operatorname{tr}\left[\frac{2 M^{2}}{-\partial^{2}+M^{2}} G_{S}^{2}\right]}{Z_{S}} \\
& m_{H}(\mu) \sim 2 m_{t}(\mu)
\end{aligned}
$$

We re-emphasise that the parameters of the Lagrangian we derive are given at the scale $\mu$, considerably below the scale of the more basic theory at $\Lambda$.

It is now a straightforward, if somewhat tedious exercise to derive the vector boson action up to second order in derivatives. The algebra is simplified if one wishes to obtain only the terms of leading order in the limit that $\Lambda$ becomes large (recall that the renormalisation factor $Z_{H} \sim \ln (\Lambda / \mu)$, is diverging logarithmically as $\left.\Lambda \rightarrow \infty\right)$. The surviving terms in this limit are:

$$
\begin{aligned}
I & =\frac{1}{3} \operatorname{tr}\left\{\left(\frac{1}{k^{2}+M^{2}}\right)^{2} \frac{1}{4}\left[Y F_{\mu \nu}(B)+P_{L} F_{\mu \nu}(W)\right]^{2}\right\} \\
& -\frac{1}{4} \operatorname{tr}\left\{\left(\frac{1}{k^{2}+M^{2}}\right)\left(Y B_{\mu}+P_{L} W_{\mu}\right)^{2}\right\} \\
& -\frac{1}{4} \operatorname{tr}\left\{\left(\frac{1}{k^{2}+M^{2}}\right)^{2}\left(Y B_{\mu}+P_{L} W_{\mu}\right) M^{2}\left(Y B_{\mu}+P_{L} W_{\mu}\right)\right\} \\
+ & \frac{1}{4} \operatorname{tr}\left\{\left(\frac{1}{k^{2}+M^{2}}\right)^{2}\left[\frac{1}{4}\left[\gamma_{\mu} W_{\mu}, M\right]^{2}-\left[\gamma_{\mu} B_{\mu}\left(y_{r}-y_{l}\right)-\frac{1}{2}\left\{\gamma_{\mu} W_{\mu}, M\right\}\right]^{2}\right]\right\}
\end{aligned}
$$

Where $W_{\mu}=W_{\mu} \cdot \tau$. In this result integrals over four dimensional $k$ and $x$ are understood. The symbol $F_{\mu \nu}(W)$ is the full, nonabelian field strength tensor. Apart from those mass terms which are not proportional to $M^{2}$, these teims are just the gauge field sector of the 
standard model with spontaneous symmetry breaking by the Higgs mechanism. The additional fine tuning, discussed above, will be necessary to guarantee that the hard mass terms in (3.7) cancel against the classical quadratic terms which were introduced into the action to cancel the four-fermion interaction. The analysis of the quadratic terms is somewhat subtle, and we present and discuss these terms in full detail in the next section.

\section{Quadratic Vector Boson Action}

All terms quadratic in boson fields arise from second order in the expansion of the logarithm and may be written:

$$
I^{(2)}=\sum_{a, b} \frac{1}{2} \operatorname{Tr} \ln \left[\frac{1}{i \not \partial+M} \Omega_{a} \frac{1}{i \not \partial+M} \Omega_{b}\right],
$$

with the term $I_{S S}^{(2)}$ already displayed. Not unexpectedly, we set:

$$
G_{B} \alpha=G_{W} \beta=E
$$

with $E$ a dimensional electric charge, together with the usual rotation in the abelian subsector:

$$
\begin{aligned}
& B_{\mu}=\alpha A_{\mu}+\beta Z_{\mu} \\
& W_{\mu}^{3}=\beta A_{\mu}-\alpha Z_{\mu}
\end{aligned}
$$

We present only the final results for the fields $W_{ \pm}, A$, and $Z$. These are:

$$
\begin{gathered}
I_{A A}^{(\mathrm{m})}=\left[\frac{1}{2}+G_{W}^{2} \beta^{2} \sum_{i} 2 Q_{i}{ }^{2} \operatorname{tr}\left[\frac{\frac{1}{2} \partial^{2}-m_{i}{ }^{2}}{\left(-\partial^{2}+m_{i}{ }^{2}\right)^{2}}\right]\right] A_{\sigma}{ }^{2} \\
I_{A A}^{(\mathrm{K} . \mathrm{E} .)}=G_{W}^{2} \beta^{2} \sum_{i} 2 Q_{i}{ }^{2} \operatorname{tr}\left[\frac{1}{6} \frac{\left(\partial^{2}\right)^{2}}{\left(-\partial^{2}+m_{i}{ }^{2}\right)^{4}}-\frac{1}{4} \frac{m_{i}{ }^{2} \partial^{2}}{\left(-\partial^{2}+m_{i}{ }^{2}\right.}+\frac{1}{4} \frac{m_{i}{ }^{2}}{\left(-\partial^{2}+m_{i}{ }^{2}\right)^{3}}\right] A_{\sigma \lambda}{ }^{2} \\
I_{Z A}^{(\mathrm{m})}=\sum_{i} \frac{1}{4} C_{i} \operatorname{tr}\left[\frac{\frac{1}{2} \partial^{2}-m_{i}{ }^{2}}{\left(-\partial^{2}+m_{i}{ }^{2}\right)^{2}}\right] Z_{\sigma} A_{\sigma} \\
I_{Z A}^{(\mathrm{K} . \mathrm{E} .)}=\sum_{i} \frac{1}{4} C_{i} \operatorname{tr}\left[\frac{1}{6} \frac{\left(\partial^{2}\right)^{2}}{\left(-\partial^{2}+m_{i}{ }^{2}\right)^{4}}-\frac{1}{4} \frac{m_{i}{ }^{2} \partial^{2}}{\left(-\partial^{2}+m_{i}{ }^{2}\right)^{4}}+\frac{1}{4} \frac{m_{i}{ }^{2}}{\left(-\partial^{2}+m_{i}{ }^{2}\right)^{3}}\right] Z_{\sigma \lambda} A_{\sigma \lambda} \\
I_{Z Z}^{(\mathrm{m})}=\left[\frac{1}{2}+\sum_{i}\left\{\frac{1}{8} R_{i} \operatorname{tr}\left[\frac{\partial^{2}}{\left(\left(-\partial^{2}+m_{i}{ }^{2}\right)^{2}\right.}\right]-\frac{1}{2} T_{i} \operatorname{tr}\left[\frac{m_{i}{ }^{2}}{\left(-\partial^{2}+m_{i}{ }^{2}\right)^{2}}\right]\right\}\right] Z_{\sigma}{ }^{2} \\
I_{Z Z}^{(\mathrm{K} . \mathrm{E} .)}=\sum_{\mathrm{i}} \operatorname{tr}\left[\left(\frac{1}{6} \frac{\left(\partial^{2}\right)^{2}}{\left(-\partial^{2}+m_{i}{ }^{2}\right)^{2}}+\frac{1}{4} \frac{m_{i}{ }^{2}}{\left(-\partial^{2}+m_{i}{ }^{2}\right)^{3}}\right) R_{i}-\frac{1}{8} \frac{m_{i}{ }^{2} \partial^{2}}{\left(-\partial^{3}+m_{i}{ }^{2}\right)^{4}} T_{i}\right] Z_{\sigma \lambda}{ }^{2}
\end{gathered}
$$




$$
\begin{aligned}
I_{W W}^{(\mathrm{m})}= & {\left[1+G_{W}^{2} \sum_{g}\left[\frac{3}{2} \operatorname{tr}\left[\frac{\partial^{2}}{\left(-\partial^{2}+m_{t_{\theta}}^{2}\right)\left(-\partial^{2}+m_{b_{g}}^{2}\right)}\right]\right.\right.} \\
+ & \left.\frac{1}{2} \operatorname{tr}\left[\frac{\partial^{2}}{\left(-\partial^{2}+m_{e_{g}}^{2}\right)\left(-\partial^{2}+m_{\nu_{g}}^{2}\right)}\right]\right] W_{\mu}^{+} W_{\mu}^{-} \\
I_{W W}^{(\text {K.E.) }}= & \frac{1}{6} G_{W}^{2} \sum_{g}\left[3 \operatorname{tr}\left[\frac{\left(\partial^{2}\right)^{2}}{\left(-\partial^{2}+m_{t_{g}}^{2}\right)^{2}\left(-\partial^{2}+m_{b_{\theta}}^{2}\right)^{2}}\right]\right. \\
& \left.+\operatorname{tr}\left[\frac{\left(\partial^{2}\right)^{2}}{\left(-\partial^{2}+m_{e_{g}}^{2}\right)^{2}\left(-\partial^{2}+m_{\nu_{g}}^{2}\right)^{2}}\right]\right] W_{\sigma \lambda}^{+} W_{\sigma \lambda}^{-}
\end{aligned}
$$

The sums in the W-W terms are over generations only, the remaining traces are over Dirac matrices and momentum, and the anti-symmetric symbols $A_{\sigma \lambda}$ are just the linear terms in the covariant field tensors:

$$
A_{\sigma \lambda}=\left(\partial_{\sigma} A_{\lambda}-\partial_{\lambda} A_{\sigma}\right)
$$

The symbols $Q_{i}, C_{i} ; R_{i}, T_{i}$, are defined by:

$$
\begin{gathered}
Q_{i}=\frac{y_{L i}}{2}+\frac{\tau_{3 i}}{2}=\frac{y_{R i}}{2} \\
C_{i}=2 \alpha \beta G_{W} G_{B}\left(y_{L i}^{2}+y_{R i}^{2}\right)-2 \alpha \beta G_{W}^{2} \tau_{3 i} \tau_{3 i}+2 G_{B} G_{W}\left(\beta^{2}-\alpha^{2}\right) \tau_{3 i} y_{L i} \\
R_{i}=G_{B}^{2} \beta^{2}\left(y_{L i}^{2}+y_{R i}^{2}\right)-2 G_{B} G_{W} \alpha \beta y_{L i} \tau_{3 i}+G_{W}^{2} \alpha^{2} \tau_{3 i} \tau_{3 i} \\
T_{i}=G_{B}^{2} \beta^{2} y_{R i} y_{L i}-G_{B} G_{W} \alpha \beta y_{R i} \tau_{3 i}
\end{gathered}
$$

Performing sums over all the fermions we find also

$$
\begin{aligned}
& \sum_{i} C_{i}=16 n_{g} G_{W}^{2} \frac{\beta}{\alpha}\left(\frac{8}{3} \beta^{2}-1\right) \\
& \sum_{i} R_{i}=8 n_{g} G_{W}^{2}
\end{aligned}
$$

where $n_{g}$ is the number of generations. As we have noted above, considerable simplification would result if only terms of leading order in the limit $\Lambda \rightarrow \infty$ were retained, were it not for the very important requirement that the photon mass must, to all intents and purposes, vanish identically, certainly also to several non-leading orders as well as leading order in the large cutoff expansion. First we note a central result, which emerges from a consideration of the hard mass terms in Equations(4.1-9). We reiterate that the hard mass terms are just those proportional to $\Lambda^{2}$. In particular one finds:

$$
I_{Z, A} \sim \Lambda^{2} G_{W}^{2}\left(\frac{5}{3} \beta^{2}-\alpha^{2}\right)
$$


with leading $\ln (.1)$ terms vanishing identically. Thus the condition:

$$
\sum_{i} C_{i}=0
$$

removes such terms from this off-diagonal action, and the next order are not present. Remarkably, this same condition, in effect $\sin ^{2}\left(\theta_{W}\right)=\frac{3}{8}$ as in the SU(5) GUT [13] model, removes the gauge non-invariant mass terms for the $Z, W$, and $A$ fields. Given this relation. the $\Lambda^{2}$ terms in the photon mass are eliminated, provided a simple consistency condition, in effect a second gap equation, is satisfied. To order $\Lambda^{2}$ this condition is:

$$
1=n_{c} \frac{G_{W}^{2} \beta^{2}}{4 \pi^{2}} \sum_{i} 2 Q_{i}{ }^{2} \Lambda^{2}=\frac{2 n_{g}}{4 \pi^{2}} G_{W}^{2} \Lambda^{2}
$$

with $n_{g}$ the number of fermion generations. The $\ln (\Lambda)$ terms in $I_{Z Z}^{(M)}$ and $I_{Z A}^{(m)}$ are automatically eliminated. To this order one then obtains predictions for the $W$ and $Z$ masses at the scale $\mu$ in terms of the top quark mass. The dimensionless couplings $g_{1}$, and $g_{2}$ of the $S U(2)$ and $U(1)$ sectors may also be identified, though not unambiguously, as we have discussed above. One finds:

$$
\frac{g_{2}}{2}=\frac{G_{W}}{\sqrt{Z_{W}}}, \quad \frac{g_{1}}{2}=\frac{G_{B}}{\sqrt{Z_{B}}}
$$

One can demonstrate the possibility of setting the photon mass to zero most simply in the somewhat artificial case of equal quark and lepton masses $m_{i}(\mu)=m(\mu)$. wen, the choice $\sum_{i} C_{i}=0$ leads to the vanishing of $I_{Z A}^{K . E .}$, and $I_{Z A}^{m}$. Both the kinetic energy and the mass matrices in the $Z, A$ sector are diagonal and one now requires more generally:

$$
1=\frac{2 G_{W}^{2}}{4 \pi^{2}} n_{g}\left(\Lambda^{2}-\mu^{2}\right)+\frac{2}{4 \pi^{2}} G_{W}^{2} \beta^{2} \sum_{i}\left(\frac{m_{i}^{4}}{m_{i}^{2}+\mu^{\prime 2}}-\frac{m_{i}^{4}}{m_{i}^{2}+\Lambda^{2}}\right) Q_{i}{ }^{2}
$$

with as stated earlier, $\ln (\Lambda)$ terms not present. Equation (4.17) simply defines $G_{W}^{2}$ as a function of $\Lambda$, say in the form:

$$
\frac{2 G_{W}^{2} \Lambda^{2} n_{g}}{4 \pi^{2}}=1+a(\Lambda)
$$

and in a fashion analogous to the gap equation (3.1). To lowest order $G_{W}^{2}=\frac{1}{2} \frac{n_{c}}{n_{g}} G_{t}^{2}$. Imposing (4.17) adds corrections to the $Z$ and $W$ masses, but as is not hard to establish, they are small. Should masses $m_{i}$ be unequal, one must first diagonalise the matrix of wave function renormalisations $[Z]$, rescale the resulting $Z$ and $A$ fields and finally rediagonalise the mass matrix. This will require that $\beta^{2}$ and $G_{W}^{2}$ both be functions of $\Lambda$ in order that mass matrix is diagonal and the photon mass zero. This will be detailed in further work, but as we have indicated would not substantially alter the results. 
We now finally quote these results with the loop integrations running from $\mu$ to $\Lambda$ :

$$
\begin{aligned}
& \mathcal{M}_{Z}^{2}=\sum_{i} \operatorname{tr}\left[\frac{\left(\frac{1}{4} R_{i}-\frac{1}{2} T_{i}\right) m_{i}^{2}}{\left(-\partial^{2}+m_{i}^{2}\right)^{2}}\right] \\
& Z_{Z}=\sum_{i} \operatorname{tr}\left[\frac{1}{6} \frac{\left(\partial^{2}\right)^{2}}{\left(-\partial^{2}+m_{i}^{2}\right)^{4}}\right] \frac{1}{4} R_{i}
\end{aligned}
$$

We find

$$
M_{Z}^{2}(\mu)=\frac{3}{5} M_{t}^{2}(\mu)=\frac{\mathcal{M}_{Z}^{2}}{Z_{Z}}
$$

ignoring the mass difference, in (4.19), and perhaps $20 \%$ less for reasonable mass choices $m_{i}(\mu)$. Similarly one finds, evaluating $Z_{W}$, and the element $M_{W W}$ of the mass matrix from $\operatorname{Eqs}(4 \cdot 10,4 \cdot 11)$ :

$$
M_{W}^{2}(\mu)=\frac{3}{8} m_{t}^{2}(\mu)
$$

In either case, to the degree of accuracy required in these preliminary computations one has for the weak angle at $\mu$ :

$$
\cos ^{2}\left(\theta_{W}\right)=\frac{M_{W}^{2}}{M_{Z}^{2}}=\frac{5}{8}
$$

This value for the weak mixing angle is that predicted by grand unified theories [13], but the intermediate scale $\mu$ at which the present calculation achieves it is not necessarily a point of equality of couplings, that is, the scale $\mu$ need not be the grand unified scale.

\section{Renormalisation Group Evolution of the Mass Ratios.}

The effective lagrangian so far defined for the scalar-vector boson sector at $\mu$ certainly seems to be that of the standard model. We now imagine the strong interactions to be simply married to the electro-weak theory, and the relationships so far obtained evolved down to, say, the scale $M_{W}=80.7 \mathrm{GeV}$. Reasonable quantities to consider are the mass ratios $\frac{M_{f}^{2}}{M_{w}^{2}}(\mu)$, and the weak mixing angle, both of which should be somewhat more free of the ambiguities discussed above than the individual couplings $g_{i}(\mu)$. We will choose the scale $\mu$ such that $\sin ^{2}\left(\theta_{W}\right)$ evolves down to the experimental value: 0.232 [14].

This choice of the intermediate scale $\mu$ at which the standard model emerges is not well-defined, and the abrupt joining of the standard model to the theory above $\mu$ resulting from the use of the values $\frac{M_{\psi}^{2}}{M_{z}^{2}}(\mu), \frac{M_{*}^{2}}{M_{i}^{2}}(\mu)$ as boundary conditions for the renormalisation group (RG) equations is probably unrealistic. However, we choose to do just this, hopefully compensating for the vagueness by fitting $\mu$, through the evolution of $\sin ^{2}\left(\theta_{W}\right)$. To leading 
order we have [10]:

$$
\begin{aligned}
\sin ^{2}\left(\theta_{W}\right)\left(M_{W}\right) & =\frac{\alpha\left(M_{W}\right)}{\alpha_{2}\left(M_{W}\right)} \\
& =\frac{3}{8}\left[1-\frac{109}{18} \frac{\alpha\left(M_{W}\right)}{\pi} \ln \frac{\mu}{M_{W}}\right]+\cdots
\end{aligned}
$$

There results $\mu=7.5 \times 10^{12} \mathrm{GeV}\left(\mu=3.7 \times 10^{14} \mathrm{GeV}\right)$ for $\sin ^{2}\left(\theta_{W}\right)\left(M_{W}\right)=.232(.210)$ $[14,2]$, the former being the most recently accepted value of the weak angle. The lower value of $\mu$ is somewhat below the usually accepted grand unified scale but still very high. Using this information it is now possible to evolve the ratio $\frac{M_{t}^{2}}{M_{W}^{2}}$. The relevant expressions are:

$$
\frac{2 \kappa_{t}}{\alpha_{2}}=\frac{4 g_{t}^{2}}{g_{2}^{2}}=\frac{M_{t}^{2}}{M_{W}^{2}}
$$

and

$$
\kappa_{t}=\frac{2 \alpha_{3}^{8 / 7}}{C+9 \alpha_{3}^{1 / 7}}
$$

The latter is one of a general family of solutions for the fermion coupling RG equation found by (KSZ) [15] but ignores electro-weak corrections at the scale $\mu$ [9]. The arbitrary parameter $C$ may be determined by first evolving $\alpha_{3}$ to $\mu$ and then using (5.2) and (5.3). One needs also, the evolution equations for $\alpha_{2}(\mu)$ and $\alpha_{3}(\mu)$ which we again take at the one-loop level $[9,10,14]$. For the ratio $\frac{M_{t}^{2}}{M_{w /}^{2}}=\frac{8}{3}$ obtained ignoring quark mass differences in the $W^{+}$wave function renormalisation (but not in the mass coefficient), we find:

$$
M_{t}\left(M_{w}\right)=2.04 M_{W}=164 \mathrm{GeV}
$$

for both scaies $\mu$ mentioned above, an apparently stable prediction. Allowing for reasonably chosen unequal quark masses in $Z_{W}$ and $Z_{Z}$ yields as mentioned above $\frac{M_{W}^{2}}{M_{i}^{2}}=\frac{3}{8} \times \frac{1}{1.18}$, and a somewhat lower $M_{t}=158 \mathrm{GeV}$ at $\mu=7.5 \times 10^{12} \mathrm{GeV}$. We have of course left out electroweak corrections and higher loops in this very preliminary work.

The Higgs mass at the scale $\mu$ is as in all NJL theories placed very near $m_{H}=2 m_{t}$. Assuming a weakly coupled Higgs evolved by the usual scalar self-coupling relation:

$$
\lambda(\mu)=\frac{\lambda\left(M_{w}\right)}{1-\frac{3 \lambda}{2 \pi^{2}} \ln \left(\frac{\mu}{M_{w}}\right)}
$$

one finds $M_{H}=139(132) \mathrm{GeV}$ and $\sin ^{2}\left(\theta_{W}\right)=.232(.21)$. This value, however, is almost certainly too low, since the Higgs mass is subject to strong corrections from coupling to $g_{t}$. Approximately including the coupling to $g_{t}$, but not to electro-weak, by assuming $\kappa_{t} \sim$ constant, and varying this constant from an average value to its value at $M_{W}$ produces 
$M_{H}=132-143 \mathrm{GeV}$ for $\sin ^{2}\left(\theta_{W}\right)=.232$ and $M_{H}^{2}=\frac{32}{3} M_{W}^{2}$ at $\mu$. But it is almost certainly true that the barely bound Higgs at $\mu$ will evolve to a more deeply bound composite at . $I_{W}$. just as pointed out by Bardeen, et. al. [2]:

\section{Conclusions.}

It seems possible to recover the standard electro-weak theory from a four-fermion theory at a scale $\Lambda \sim 10^{13}-10^{15} \mathrm{GeV}$, perhaps somewhat below the usual grand unification scule. The quark-meson sector, incidentally, is consistently defined by setting $G_{W} W=g_{W} / 2 \tilde{W}$, that is $g_{W}=G_{W} /\left(2 \sqrt{Z_{W}}\right)$, etc.. No deeper basis is given for the four-fermion theory, nor for the asymmetric coupling of quarks to the mass-generating scalar field. One might imagine that the theory arises from massive vector bosons at this upper scale, with the observed $W$, and $Z$ arising as very light composite images of their higher mass analogues. A more natural reason for the gauge symmetry at the present experimental mass scale would then emerge.

The treatment we present here follows in many ways that outlined in [2], but of course, we have added a specific $S U(2) \times U(1)$ current-current interaction to the four-fermion theory. In both presentations the four-fermion theory is expected to be valid at some scale: we have introduced an intermediate scale $\mu, \Lambda \gg>\mu>M_{w}$, at which the four-fermion action apparently yields the standard $S U(2) \times U(1)$ theory with very simple expressions for the lagrangian parameters. At this scale, the coupling $\sim\left(\ln \frac{\Lambda^{2}}{\mu^{2}}\right)^{-1}$ is not very small, but still hopefully perturbative. It is surely, then, somewhat crude to turn on the full Standard model at $\mu$ and evolve downward. Just as with Bardeen et. al., the NJL wave function renormalisation $Z(\mu) \sim \ln \left(\frac{\Lambda}{\mu}\right)$ vanishes at $\mu \rightarrow \Lambda$, where indeed they initiate the matching.

Introducing extra generations with still one high mass (up) quark dominating would leave $\sin ^{2}\left(\theta_{W}\right)$, and $\frac{M_{w}^{2}}{M_{t}^{2}}$ unchanged at $\mu$, but would weaken the couplings at the scale $\mu$ as well as altering the evolutions upwards to $\mu$. Moreover the altered mass prediction would then be for this new up quark, and presumably the top would be lower.

Finally, it should be pointed out that with all the four-fermion couplings inversely proportional to the cutoff, it should be possible to investigate multi-fermion loop contributions and check the perturbative nature of the four-fermion interaction. 


\section{Acknowledgements}

The authors are extremely grateful to Bill Marciarı for encouragement, and invaluable clarifying instruction, without which it would have been difficult to complete this work, and for specific suggestions about for example the inclusion of extra generations. They are also grateful to Frank Paige for discussions on high mass scales and the meaning of the "utoff in non-renormalisable theories. 


\section{References}

[1] Y. Nambu, Fermi Institute Preprint 89-08 (1989).

[2] W. Bardeen, C. T. Hill, M. Lindner, Fermi-PUB-89/127-T.

[3] V. A. Miranski, M. Tanabashi, K. Yamawaki, Mod. Phys. Lett. A4, 1043 (1989).

[4] P. W. Higgs, Phys. Lett. 12.132 (1964).

[5] S. Weinberg, Phys. Rev. Lett. 191264 (1967);

A. Salam, in Elementary Particle Theory; Relativistic Groups, and Analyticity.

Edited by N. Svartholm, Proceedings of the Nobel Symposium \#8 (Almqvist, Wiksell, Stockholm 1968);

S. L. Glashow, J. Iliopulos, L. Maiani, Phys. Rev. D2 1285 (1970).

[6] Y. Nambu \& G. Jona-Lasinio Phys. Rev. 122,\#1 (1961) p.345.

[7] D. E. Kahana, U. Vogl, in preparation.

[8] R.D. Ball, Invited talk, given at "Skyrmions and Anomalies", Mogillany, Poland, Feb. 21-23, 1987, to be published in the conference proceedings;

A. Dhar, R. Shankar, S.R. Wadia, Phys. Rev. D31 \#12 (1985).

Ebert \& Reinhardt, Nucl. Phys. B271 (1986) p.188.

[9] W. Marciano, Phys. Rev. Lett. 622793 (1989);

Phys. Rev. Lett. 71219 (1990).

[10] W. Marciano, A. Sirlin, Phys. Rev. D22 2695 (1980);

M. Goldhaber, W. Marciano, Comments in Nuclear and Particle Physics.

[11] D. E. Kahana Phys. Lett. B229 9 (1989).

[12] D. Gross \& J. Neveu, Phys. Rev. D10,\#10 (1974) p.3235.

[13] H. Georgi, S. Glashow, Phys. Rev. Lett. 32438 (1974);

H. Georgi, H. Quinn, and S. Weinberg, Phys. Rev. Lett., 33451 (1974).

[14] S. Fanchiotti, and A. Sirlin Phys. Rev. D41 319 (1990).

[15] J. Kubo, K. Sibold, and W. Zimmermann, Phys. Lett. B220 185 (1989). 

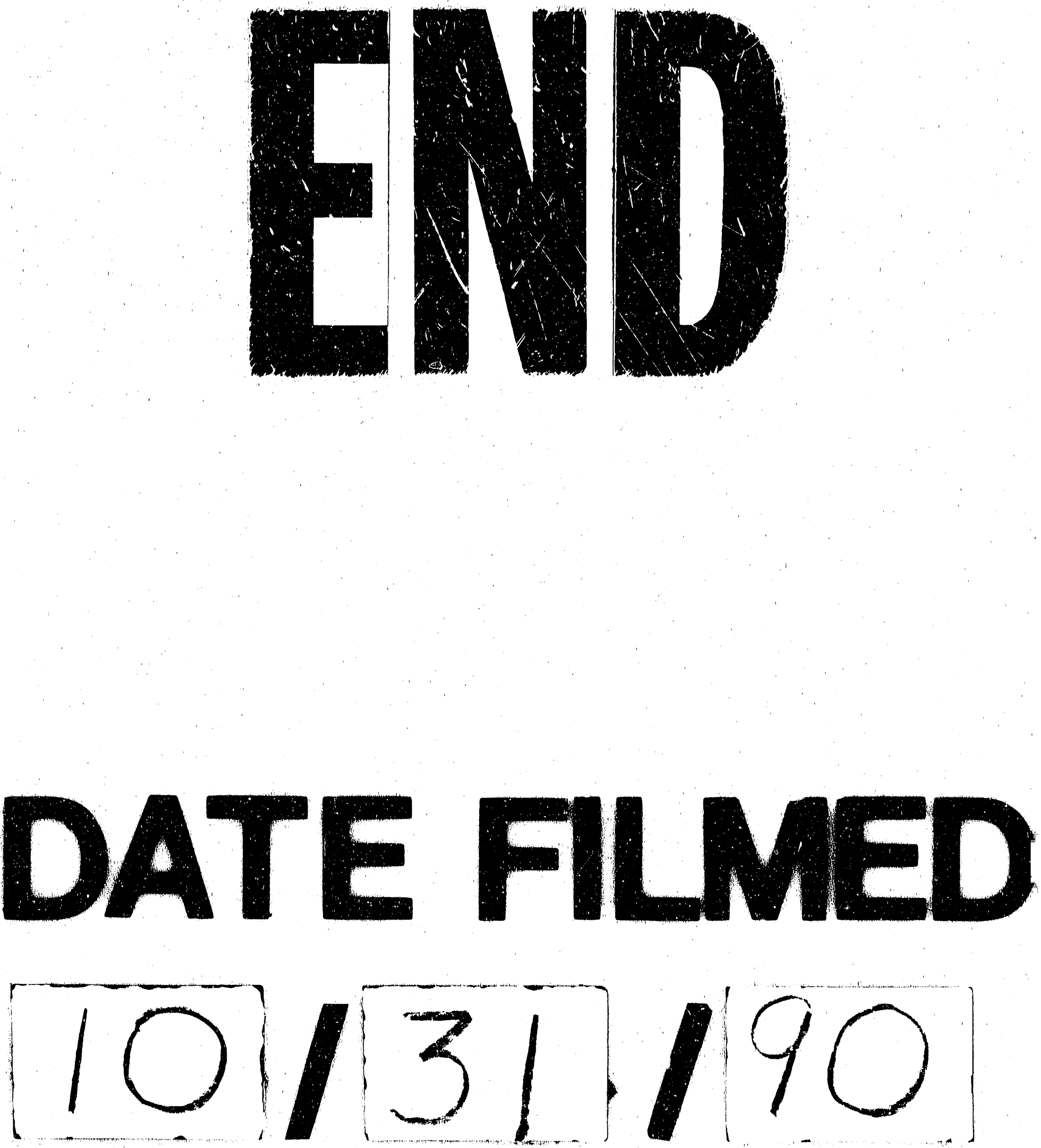
